CLINICAL STUDY

\title{
Analysis of extended human leukocyte antigen haplotype association with Addison's disease in three populations
}

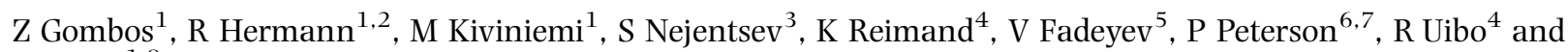 \\ J Ilonen ${ }^{1,8}$ \\ ${ }^{1}$ Immunogenetics Laboratory, University of Turku, Medicity, Tykistökatu 6 A 4th, 20520 Turku, Finland, ${ }^{2}$ Immunogenomics Laboratory, CellScreen \\ Applied Biomedical Research Center, Semmelweis University, Budapest, Hungary, ${ }^{3}$ Juvenile Diabetes Research Foundation/Wellcome Trust Diabetes and \\ Inflammation Laboratory, Cambridge Institute for Medical Research, University of Cambridge, Cambridge, UK, ${ }^{4}$ Immunology Group, Institute of General \\ and Molecular Pathology, University of Tartu, Tartu, Estonia, ${ }^{5}$ Department of Endocrinology, Moscow Medical Academy, Moscow, Russia, ${ }^{6}$ Institute of \\ Medical Technology, University of Tampere, Tampere, Finland, ${ }^{7}$ Molecular Pathology Group, Institute of General and Molecular Pathology, University of \\ Tartu, Tartu, Estonia and ${ }^{8}$ Department of Clinical Microbiology, University of Kuopio, Kuopio, Finland
}

(Correspondence should be addressed to Z Gombos; Email: zsogom@utu.fi)

\begin{abstract}
Objective: Addison's disease is an organ-specific autoimmune disorder with a polygenic background. The aim of the study was to identify non-class II human leukocyte antigen (HLA) susceptibility genes for Addison's disease.

Design and methods: Addison's disease patients from three European populations were analysed for selected HLA-DR-DQ alleles and for 11 microsatellite markers covering $\sim 4 \mathrm{Mb}$ over the HLA region. Subjects were 69 patients with Addison's disease from Estonia (24), Finland (14) and Russia (31). Consecutively recruited healthy newborns from the same geographical regions were used as controls (269 Estonian, 1000 Finnish and 413 Russian). Association measures for HLA-DRB1, DQB1, DQA1 and 11 microsatellites between D6S273 and D6S2223 were taken. A low-resolution full-house typing was used for HLA class II genes, while microsatellite markers were studied using fluorescence-based DNA fragment sizing technology. Results: We confirmed that the HLA-DR3-DQ2 and the DQB1*0302-DRB1*0404 haplotypes confer disease susceptibility. In Russian patients, we also found an increase of DRB1*0403 allele, combined with DQB1*0305 allele in three out of six cases $(P<0.0001)$. Analysis of 11 microsatellite markers including STR MICA confirmed the strong linkage in DR3-DQ2 haplotypes but DRB1*0404-DQB1*0302 haplotypes were diverse. MICA5.1 allele was found in 22 out of 24 Estonian patients, but results from Finnish and Russian patients did not support its independent role in disease susceptibility. Conclusion: HLA-DRB1*0403 was identified as a novel susceptibility allele for Addison's disease. Additionally, we found no evidence of a non-class II HLA disease susceptibility locus; however, the HLADR3-DQ2 haplotype appeared more conserved in patient groups with high DR-DQ2 frequencies.
\end{abstract}

European Journal of Endocrinology 157 757-761

\section{Introduction}

Addison's disease is an organ-specific autoimmune disease characterized by the damage of adrenal cortex and insufficiency of corticosteroid production (1). Steroidogenic P450 cytochrome enzymes have been identified as target antigens of the autoimmune response $(2,3)$ and circulating antibodies against 21-hydroxylase are a sensitive diagnostic marker of the disease with a tendency to decrease in percentage when adrenal gland destruction progresses (4). The disease is often found as a part of polyendocrinopathy syndromes combining it with other organ-specific autoimmune diseases, thyroid disease and type 1 diabetes (T1D) being the most frequent associates (5).

Human leukocyte antigen (HLA) association of the disease with the common 'autoimmune' HLA-DR3 haplotype has been known for decades (6) and some studies have also found an increased frequency of HLA-DR4 among patients $(7,8)$. An association with specific HLA-DR4-positive haplotype, HLA-DQB1*0302DRB1*0404, was described among Addison's disease patients and diabetic children positive for 21-hydroxylase autoantibodies (9). More recently, analysis of microsatellites as well as some candidate genes within class I region in Addison's disease (10) and other autoimmune diseases (11) has given a basis to suggest that there might be other susceptibility genes outside of DR-DQ region. Among the candidates for association with Addison's disease is the major histocompatibility complex class I polypeptide-related sequence A (STR MICA), of which allele MICA5.1 was reported to be strongly associated with Addison's disease (12) or progression to Addison's disease among diabetic persons with antibodies against 21-hydroxylase (13). 
To further explore the HLA-associated susceptibility, we analysed Addison's disease patients from three European populations for selected HLA-DRB1 and HLA-DQB1 alleles and for 11 microsatellite markers covering $\sim 4 \mathrm{Mb}$ over the HLA region.

\section{Materials and methods}

Sixty-nine Addison's disease patients, including 24 from Estonia, 14 from Finland and 31 from Russia, were studied. Most of the patients were adults (66 out of 69). None of these patients had type 1 polyendocrinopathy syndrome but type 2 autoimmune polyendocrinopathy syndrome was present in 20 out of 69 patients (29.0\%). The 21-hydroxylase antibodies were measured by radiobinding assay as described elsewhere (14). Altogether, $70 \%$ of studied patients from Estonia and Finland were antibody positive, including seven out of eight patients with type 2 autoimmune polyendocrinopathy syndrome. We have further clinical information only from Finnish and Estonian patients. Autoimmune polyglandular syndromes were present in six Finnish and two Estonian patients. No reasonable further analyses can be based on this incomplete information. As a control we used background population frequencies of the class II haplotypes established in a previous study of the same populations (15).

For genotyping of the HLA class II genes HLA-DRB1, HLA-DQA1 and HLA-DQB1, we used a panel of oligonucleotide probes designed for low-resolution fullhouse typing, including a more detailed genotyping of the HLA-DR4 subtypes (16). Microsatellite markers (D6S273, TNFa, C12A, STR MICA, MIB, C125, C143, C245, C3211, MOGc and D6S2223) were studied using PCR primers labelled with fluorescent dyes (17) and products were identified by fluorescence-based automated DNA sequencing according to the manufacturer's instructions (MegaBace 1000). Commonly referred allele names were used for the TNFa and STR MICA microsatellites. For the other microsatellite loci, allele numbers corresponded to the length of the PCR product (estimated by sequencing of homozygous samples).

\section{Results}

Table 1 shows haplotype frequencies among patients and controls in each population. The HLA-DR3 haplotype was associated with Addison's disease in all analysed populations but there was a considerable variation in the strength of this association. Eleven out of $14(78.6 \%)$ of Finnish patients were positive for this haplotype when compared with 12 out of $24(50.0 \%)$ Estonian and only 11 out of 31 (35.5\%) Russian patients $(P=0.03 ; \mathrm{df}=2)$. In addition to the increased frequency of HLA-DR3 haplotype, we also found a significant increase in DQB1*0302-DRB1*0404 haplotype among Estonian and Russian patients $(P=0.0056$ and 0.0051 respectively). In the Finnish population, this haplotype was also more common among patients than controls, although the difference did not quite reach statistical significance.

Among Russian patients, we also found that the DRB1*0403 allele was significantly more common than among controls ( 6 out of 31 and 2 out of 413 respectively, $P<0.0001)$. It was present in six patients, in three cases conventionally with DQB1*0302 and in three cases combined with the DQB1*0305 allele. Results of microsatellite analysis are shown in Fig. 1 together with individual HLA-DQB1 and HLA-DQA1 genotypes. Microsatellite markers present on both chromosomes are shown. Markers are ordered from centromere to telomere and sorted to demonstrate the DQB $1{ }^{*} 02-\mathrm{DQB} 1{ }^{*} 05$ (DR3-DQ2) haplotype and microsatellite markers characterizing it. The presentation also offers a way to analyse the possible contribution to disease risk by the other haplotype. The allele associated with the extended haplotype in each locus is marked using shading. We see the known strong conservation of 8.1 haplotype in the class III region but not in the telomeric class I region, which is most pronounced in Finnish samples. All 11 haplotypes share D6S273 and TNFa alleles, two differ at

Table 1 Distribution of Addison's disease-associated haplotypes of the HLA class II genes in Estonians, Finns and Russians.

\begin{tabular}{|c|c|c|c|c|c|c|}
\hline Population & Haplotype & Patients $N(\%)$ & Controls $N(\%)$ & OR & $\boldsymbol{P}$ & $95 \% \mathrm{Cl}$ \\
\hline \multirow{4}{*}{ Finns } & DR3, DQB1*02-DQA1*05 & $11(78.6)$ & $202(20.2)$ & 14.5 & $<0.0001$ & $3.7-41.4$ \\
\hline & DR4, DQB1*0302-DRB1*0401 & $2(14.3)$ & $124(12.4)$ & - & NS & - \\
\hline & DR4, DQB1 ${ }^{*} 0302-\mathrm{DRB} 1{ }^{*} 0404$ & $3(20.0)$ & $62(5.2)$ & 4.1 & 0.078 & $0.9-16.5$ \\
\hline & Total & 14 & 1000 & & & \\
\hline \multirow{4}{*}{ Estonians } & DR3, DQB1*02-DQA1*05 & $12(50.0)$ & $60(22.3)$ & 3.5 & 0.0056 & $1.4-8.8$ \\
\hline & DR4, DQB1*0302-DRB1*0401 & $0(0)$ & $12(4.5)$ & - & NS & - \\
\hline & DR4, DQB1*0302-DRB1*0404 & 7 (29.2) & $25(9.2)$ & 4.0 & 0.008 & $1.4-11.6$ \\
\hline & Total & 24 & 269 & & & \\
\hline \multirow[t]{4}{*}{ Russians } & DR3, DQB1*02-DQA1*05 & $11(35.5)$ & $65(15.7)$ & 2.9 & 0.0102 & $1.3-6.8$ \\
\hline & DR4, DQB1*0302-DRB1*0401 & $3(9.7)$ & $31(7.5)$ & - & NS & - \\
\hline & DR4, DQB1*0302-DRB1*0404 & $7(22.6)$ & $28(6.8)$ & 4.0 & 0.0051 & $1.4-10.9$ \\
\hline & Total & 31 & 413 & & & \\
\hline
\end{tabular}

$P$ values were calculated by $\chi^{2}$-test with continuity correction. 
\begin{tabular}{|l|l|l|l|l|l|l|l|l|l|l|l|l} 
DQA1 & DQB1 & D6S273 & TNFa & C12A & STR MICA & MIB & C125 & C143 & C245 & C3211 & MOGc & D6S2223 \\
\hline
\end{tabular}

\begin{tabular}{|c|c|c|c|c|c|c|c|c|c|c|c|c|c|c|c|c|c|c|c|c|c|c|c|}
\hline \multicolumn{24}{|l|}{ Finns } \\
\hline 03,05 & 0302 & 137 & 141 & 2 & 11 & 242 & 256 & 5.1 & 1 & 336 & 350 & 94 & \begin{tabular}{|l|}
198 \\
\end{tabular} & 417 & 49 & 436 & \begin{tabular}{|l|}
436 \\
\end{tabular} & 195 & 199 & 130 & 148 & 170 & 170 \\
\hline 03,05 & 02,0302 & 135 & 141 & 2 & 6 & 236 & 256 & 4 & 5.1 & 336 & 346 & 194 & 208 & 441 & 449 & 436 & 468 & 195 & 209 & 132 & 132 & 168 & 168 \\
\hline 05 & 02,0602 & 137 & 141 & 2 & 11 & 250 & 256 & 5.1 & 5.1 & 336 & 350 & 194 & 198 & 417 & 449 & 436 & 436 & 191 & 195 & 122 & 132 & 168 & 170 \\
\hline 05 & 2,02 & 141 & 141 & 2 & 2 & 256 & 256 & 5.1 & 5.1 & 350 & 350 & 194 & 194 & 445 & 449 & 436 & 436 & 207 & 217 & 132 & 148 & 170 & 172 \\
\hline 05 & 02,04 & 135 & 141 & 2 & 6 & 242 & 256 & 5.1 & 5.1 & 332 & 350 & 194 & 194 & 449 & 449 & 428 & 436 & 209 & 217 & 122 & 148 & 170 & 170 \\
\hline 03,05 & 02,0302 & 137 & 141 & 2 & 6 & 250 & 256 & 5.1 & 5.1 & 332 & 350 & 194 & 200 & 425 & 449 & 436 & 436 & 207 & 217 & 130 & 148 & 170 & 170 \\
\hline 05 & 02,02 & 141 & 141 & 2 & 2 & 256 & 256 & 5.1 & 5.1 & 350 & 350 & 194 & 194 & 449 & 453 & 428 & 480 & 191 & 195 & 148 & 148 & 170 & 170 \\
\hline 05 & 02,0301 & 137 & 141 & 2 & 4 & 238 & 256 & 5.1 & 5.1 & 350 & 356 & 194 & 208 & 441 & 441 & 428 & 468 & 195 & 195 & 132 & 136 & 170 & 170 \\
\hline 05 & 02,0602 & 141 & 141 & 2 & 11 & 242 & 256 & 4 & . & 336 & 350 & 194 & 198 & 417 & 449 & 436 & 436 & 195 & 201 & 122 & 130 & 170 & 172 \\
\hline 05 & 02,02 & 135 & 141 & 2 & 2 & 238 & 242 & 5.1 & 5.1 & 350 & 350 & 194 & 194 & 449 & 453 & 436 & 436 & 195 & 201 & 148 & 148 & 170 & 170 \\
\hline 05 & 02,02 & 133 & 141 & 2 & 2 & 236 & 258 & 5.1 & 6 & 332 & 350 & 204 & 210 & 441 & 449 & 436 & 476 & 191 & 191 & 122 & 148 & 170 & 170 \\
\hline 03 & 0303,0501 & 131 & 135 & 5 & 11 & 236 & 256 & 4 & 9 & 350 & 352 & 210 & 218 & 441 & 445 & 428 & 480 & 187 & 191 & 130 & 132 & 170 & 170 \\
\hline 03 & 0302,0501 & 131 & 135 & 1 & 5 & 236 & 256 & 4 & , & 326 & 352 & 190 & 218 & 429 & 441 & 432 & 432 & 205 & 211 & 130 & 30 & 170 & 170 \\
\hline 03 & 04,0302 & 35 & 137 & 11 & 13 & 238 & 242 & 5.1 & 5.1 & 326 & 350 & 190 & 194 & 433 & 445 & 432 & 476 & 205 & 211 & 130 & 132 & 170 & 170 \\
\hline Estonians & & & & & & & & & & & & & & & & & & & & & & & \\
\hline 05 & 02,04 & 135 & 141 & 2 & \begin{tabular}{l|l}
13 \\
\end{tabular} & 238 & 256 & 5.1 & 5.1 & 326 & 350 & 194 & 204 & 433 & 449 & 432 & 436 & 195 & 201 & 136 & 148 & 170 & 170 \\
\hline 05 & 02,0602 & 137 & 141 & 2 & 11 & 242 & 256 & 5.1 & 5.1 & 336 & 350 & 194 & 198 & 417 & 449 & 436 & 436 & 195 & 199 & 130 & 148 & 170 & 170 \\
\hline 03,05 & 02,0302 & 135 & 141 & 2 & 11 & 246 & 256 & 5.1 & 5.1 & 350 & 356 & 194 & 204 & 449 & 449 & 428 & 436 & 195 & 195 & 130 & 148 & 168 & 170 \\
\hline 03,05 & 02,0302 & 135 & 141 & 2 & 10 & 254 & 256 & 5.1 & 9 & 332 & 350 & 194 & 208 & 421 & 449 & 436 & 436 & 195 & 211 & 122 & 148 & 170 & 170 \\
\hline 03,05 & 02,0302 & 137 & 141 & 2 & 6 & 250 & 256 & 5.1 & 9 & 332 & 350 & 194 & 200 & 425 & 449 & 436 & 436 & 195 & 195 & 130 & 134 & 170 & 170 \\
\hline 03,05 & 02,0302 & 135 & 139 & 2 & 6 & 250 & 256 & 5.1 & 9 & 350 & 356 & 194 & 200 & 425 & 449 & 436 & 436 & 195 & 195 & 132 & 134 & 170 & 172 \\
\hline 05 & 02,0602 & 135 & 141 & 2 & 11 & 242 & 256 & 5.1 & 51 & 336 & 350 & 194 & 198 & 417 & 449 & 436 & 436 & 195 & 195 & 128 & 30 & 170 & 170 \\
\hline 03,05 & 0302 & 139 & 141 & 2 & 1 & 242 & 256 & 5.1 & 5.1 & 336 & 350 & 194 & 198 & 417 & 449 & 436 & 436 & 09 & 217 & 132 & 48 & 170 & 170 \\
\hline 05 & 02,04 & 135 & 141 & 2 & 2 & 250 & 256 & 5.1 & 5.1 & 350 & 350 & 194 & 208 & 445 & 453 & 428 & 436 & 189 & 195 & 130 & 130 & 170 & 170 \\
\hline 05 & 02,0602 & 131 & 141 & 2 & 11 & 236 & 256 & 4 & 5.1 & 350 & 350 & 198 & 198 & 445 & 449 & 436 & 480 & 195 & 201 & 130 & 132 & 170 & 170 \\
\hline 05 & 02,0301 & 135 & 141 & 2 & 12 & 238 & 254 & 5.1 & 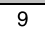 & 348 & 350 & 194 & 212 & 445 & 449 & 428 & 436 & 195 & 211 & 130 & 144 & 170 & 172 \\
\hline 03,05 & 02,0302 & 135 & 143 & 2 & 6 & 236 & 256 & 4 & 5.1 & 340 & 350 & 180 & 194 & 433 & 449 & 428 & 436 & 205 & 215 & 132 & 138 & 168 & 168 \\
\hline 0201,03 & 02,0301 & 131 & 135 & 6 & 7 & 236 & 244 & 5.1 & 5.1 & 336 & 356 & 190 & 212 & 441 & 449 & 432 & 436 & 195 & 201 & 122 & 132 & 170 & 170 \\
\hline 0201,03 & 0301 & 131 & 135 & 6 & 7 & 236 & 244 & 5.1 & 5.1 & 336 & 356 & 190 & 212 & 449 & 449 & 432 & 436 & 195 & 201 & 122 & 132 & 170 & 170 \\
\hline 05 & 0301,0602 & 137 & 137 & 4 & 11 & 238 & 242 & 5.1 & 5.1 & 336 & 356 & 194 & 210 & 417 & 449 & 436 & 472 & 205 & 209 & 132 & 136 & 170 & 172 \\
\hline 05 & 0301,0602 & 137 & 137 & 4 & 11 & 238 & 242 & 5.1 & 5.1 & 336 & 356 & 198 & 198 & 417 & 437 & 436 & 472 & 195 & 201 & 132 & 136 & 170 & 172 \\
\hline & ? & 137 & 137 & 4 & 11 & 238 & 242 & 5.1 & 5.1 & 338 & 356 & 190 & 198 & 417 & 449 & 432 & 43 & 95 & 195 & 122 & 130 & 170 & 170 \\
\hline & 0502,0602 & 137 & 137 & 11 & 11 & 238 & 242 & 5.1 & 9 & 348 & 350 & 210 & 212 & 445 & 445 & 428 & 480 & 195 & 217 & 122 & 130 & 170 & 170 \\
\hline & & 137 & 137 & 11 & 11 & 238 & 42 & 5.1 & 9 & 348 & 352 & 00 & 212 & & 15 & 428 & 480 & 99 & 207 & 22 & 130 & 170 & 170 \\
\hline 03 & 0301,0602 & 135 & 139 & 6 & 11 & 236 & 242 & 5.1 & 5.1 & 336 & 356 & 188 & 198 & 417 & 441 & 432 & 436 & 195 & 195 & 130 & 48 & 170 & 172 \\
\hline 03 & 0302,0603 & 135 & 137 & 7 & 11 & 242 & 246 & 5.1 & 5.1 & 336 & 350 & 198 & 204 & 417 & 449 & 428 & 436 & 195 & 195 & 130 & 138 & 168 & 170 \\
\hline 05 & 0301,0301 & 131 & 135 & 5 & 7 & 254 & 254 & 5.1 & 6 & 326 & 352 & 200 & 29 & 417 & 433 & 432 & 432 & 05 & 207 & 132 & 32 & 168 & 168 \\
\hline 03 & 0303,0501 & 135 & 137 & 5 & 10 & 242 & 252 & 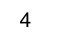 & 9 & 346 & 352 & 198 & 218 & 441 & 445 & 428 & 432 & 195 & 201 & 130 & 130 & 170 & 170 \\
\hline 05 & 0301,0301 & 135 & 135 & 10 & 13 & 238 & 238 & 5 & 9 & 332 & 34 & 0 & 208 & & 4 & 428 & & 95 & yy & 132 & 138 & 170 & 172 \\
\hline Russians & & & & & & & & & & & & & & & & & & & & & & & \\
\hline 05 & $0:$ & 137 & 141 & 2 & 11 & 242 & 256 & 5.1 & 5.1 & 338 & 35 & 14 & 19 & 417 & 49 & 436 & 436 & 35 & 195 & 32 & 148 & 168 & 170 \\
\hline 03,05 & 02 & 137 & 141 & 2 & 11 & 242 & 256 & 5.1 & 5. & 336 & 350 & 194 & 198 & 417 & 449 & 436 & 43 & 95 & 226 & 130 & 148 & 170 & 170 \\
\hline 03,05 & & 133 & 141 & 2 & 11 & 24 & 256 & 5.1 & 5.1 & 336 & 350 & 94 & 198 & 41 & 449 & 436 & 43 & 7 & 207 & 30 & 30 & 168 & 170 \\
\hline 03,05 & 0 & 135 & 141 & 2 & 11 & 248 & 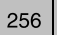 & 5.1 & 5.1 & 350 & 356 & 194 & 204 & 44 & 449 & 428 & 43 & 11 & 217 & 122 & 48 & 170 & 170 \\
\hline 03,05 & & 135 & 141 & 2 & 11 & 256 & 258 & 4 & 5.1 & 350 & 350 & 194 & 204 & 449 & 449 & 436 & 476 & 1 & 21 & 18 & 148 & 170 & 170 \\
\hline 03,05 & & 137 & 141 & 2 & 11 & 242 & 256 & 5.1 & 5.1 & 336 & 350 & 194 & 198 & 417 & 445 & 436 & 436 & 95 & 226 & 30 & 48 & 168 & 170 \\
\hline 05 & 02,0503 & 137 & 141 & 2 & 2 & 256 & 258 & 4 & 5.1 & 352 & 352 & 196 & 196 & 449 & 449 & 436 & 480 & 195 & 217 & 130 & 148 & 170 & 170 \\
\hline 03,05 & & 133 & 141 & 2 & 7 & 236 & 88 & 5.1 & - & 326 & 350 & 94 & 216 & 417 & 445 & 436 & 472 & 207 & 215 & 22 & 132 & 170 & 170 \\
\hline 03,05 & 305 & 135 & 141 & 6 & 13 & 238 & 238 & 6 & 6 & 326 & 326 & 206 & 206 & 433 & 437 & 480 & 480 & 195 & 195 & 144 & 144 & 170 & 170 \\
\hline 05 & & 129 & 13 & 5 & 7 & 236 & 254 & 6 & 6 & 326 & 342 & 212 & 218 & 417 & 437 & 432 & 432 & 195 & 195 & 122 & 132 & 168 & 170 \\
\hline 03,05 & 302 & 135 & 137 & 2 & 10 & 23 & 250 & 5.1 & $y$ & 332 & 350 & 190 & 194 & 421 & 45 & 436 & 472 & 207 & 217 & 122 & 148 & 170 & 170 \\
\hline 0201,05 & & 133 & 135 & 2 & 7 & 238 & 244 & 5 & 6 & 36 & 348 & 202 & 206 & 441 & 445 & 428 & 448 & 195 & 205 & 122 & 136 & 170 & 170 \\
\hline 0201,0 & & 131 & 14 & 2 & 7 & 244 & 256 & 5.1 & 5.1 & 350 & 350 & 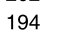 & 194 & 44 & 53 & 436 & 43 & 17 & 19 & 130 & 148 & 170 & 170 \\
\hline 0201 & 0 & 131 & 13 & 2 & 7 & 244 & 252 & 5.1 & 9 & 336 & 344 & 196 & 202 & 445 & 15 & 6 & 436 & 95 & 201 & 34 & 44 & 168 & 170 \\
\hline & & 13 & 13 & 10 & 11 & 24 & 246 & 5.1 & 5.1 & 33 & 35 & 8 & 210 & 41 & & 436 & 10 & 195 & 205 & 30 & 32 & 170 & 172 \\
\hline 0201 & & 135 & 13 & 7 & 11 & 238 & 256 & 5.1 & 6 & 336 & 342 & 198 & 202 & 41 & 433 & 436 & 476 & 09 & 21 & 30 & 136 & 170 & 170 \\
\hline 0201,0 & & 131 & 13 & 7 & 11 & 244 & 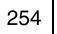 & 5.1 & 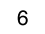 & 33 & 348 & 196 & 212 & 445 & 449 & 432 & 6 & 95 & 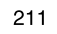 & 2 & 32 & 70 & 172 \\
\hline 0201 & 03 & 133 & 137 & 3 & 7 & 244 & 25 & 6 & 6 & 334 & 348 & 0 & 212 & 437 & 5 & 432 & 476 & 95 & 201 & 32 & 44 & 170 & 170 \\
\hline 03 & 0501 & 135 & 141 & 2 & 6 & 242 & 250 & 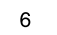 & 6 & 332 & 348 & 190 & 200 & 441 & 465 & 436 & 476 & 91 & 195 & 22 & 132 & 170 & 170 \\
\hline & & 135 & 13 & 2 & 11 & 242 & 250 & 5.1 & 5.1 & 328 & 336 & 88 & 200 & 41 & 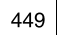 & 428 & 6 & 95 & 197 & 130 & 132 & 170 & 170 \\
\hline 03 & 2,0602 & 137 & 137 & 2 & 11 & 242 & 250 & 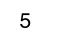 & 6 & 326 & 348 & 192 & 208 & 44 & 449 & 432 & 432 & 209 & 217 & 148 & 148 & 168 & 170 \\
\hline 03 & & 129 & 13 & 2 & . & 25 & - & 5 & 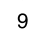 & 342 & 346 & 192 & 26 & 42 & 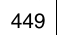 & 428 & 47 & 197 & 203 & 130 & 144 & 170 & 170 \\
\hline & 6001 & 133 & 135 & 2 & 10 & 236 & 258 & 6 & 9 & 332 & 332 & 190 & 208 & 421 & 37 & 476 & 476 & 95 & 203 & 122 & 130 & 170 & 172 \\
\hline 03,05 & 0 & 13 & 13 & 4 & 10 & 00 & 242 & 4 & 5.1 & 346 & 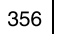 & 190 & 198 & 43 & 437 & 428 & 47 & 33 & 215 & 2 & 32 & 72 & 72 \\
\hline 05 & 1,0501 & 135 & 137 & 4 & 5 & 238 & 256 & 5.1 & 9 & 352 & 354 & 190 & 218 & 43 & 441 & 432 & 432 & 95 & 201 & 122 & 130 & 170 & 72 \\
\hline 03 & 0502 & 135 & 137 & 11 & 11 & 242 & 246 & 5.1 & 5.1 & 33 & 356 & 198 & 204 & 417 & 49 & 428 & 436 & 9 & 95 & 22 & 30 & 70 & 70 \\
\hline & & 137 & 137 & 6 & 11 & 23 & 244 & 5.1 & 6 & 332 & 338 & 198 & 200 & 41 & 25 & 436 & 36 & 05 & 11 & 0 & 32 & 168 & 170 \\
\hline 03,05 & 305 & 135 & 137 & 10 & 13 & 238 & 260 & 4 & 6 & 344 & 350 & 196 & 212 & 441 & 445 & 428 & 436 & 95 & 226 & 122 & 36 & 170 & 170 \\
\hline 05 & 01 & 137 & $13 s$ & 6 & 11 & 236 & 23 & 4 & 9 & 340 & 348 & 180 & 212 & 43 & 445 & 428 & 43 & 195 & 95 & 6 & 10 & 170 & 170 \\
\hline & 502 & 133 & 137 & 1 & 6 & 246 & 250 & 5 & - & 326 & 342 & 188 & 194 & 429 & 433 & 428 & 428 & 195 & 217 & 148 & 152 & 170 & 170 \\
\hline 03 & 0302,0302 & 35 & 35 & e & 10 & 236 & & 9 & & 332 & 48 & 88 & 212 & 37 & 10. & 28 & 472 & 195 & 207 & 22 & 130 & 168 & 170 \\
\hline
\end{tabular}

Figure 1 Extended HLA haplotypes in Finnish, Estonian and Russian patients with Addison's disease. Conserved haplotype structures are marked in grey. 
C12A and one more at STR MICA. MIB allele 350 is found again in 13 out of 14 cases but only three haplotypes seem to extend to C3211. A similar type of pattern is seen in the Estonian patients, conserved haplotypes extend even further to C3211 where 7 of the original 11 DR3 haplotypes may continue and 4 may be extended up to MOGc marker. In the Russian haplotypes, there is slightly more heterogeneity in loci adjacent to HLA-DQ, at D6S273 and TNFa locus, where 8 out of 11 patients share the common allele 2 .

Of the markers found on the haplotypes other than the DR3 haplotype, MICA5.1 is conspicuous especially on Estonian samples, less so in Finnish and Russians. In the analysis of 166 haplotypes non-transmitted in Finnish families to a diabetic child and therefore representing a background Finnish population, MICA5.1 allele was the most common allele present on $51.2 \%$ of all haplotypes (unpublished results). These haplotypes represent the Finnish background population and the results demonstrate that the high frequency of MICA5.1 allele is to be expected. Similarly, the most telomeric D6S2223 marker is hardly informative due to the high frequency of $170 \mathrm{bp}$ allele.

Similar analysis of DQB1*0302-DRB1*0404 haplotypes revealed very poor linkage disequilibrium in them. TNFa11 was the most common allele at that locus but it was found (together with linked D6S273 allele) only in one of the three Finnish, two of the seven Estonian and four of the seven Russian haplotypes. Absence of T1Dassociated DQB1*0302-DRB1*0404-B1*39 haplotype (18) was also confirmed by the lack of HLA-B*39 allele except in one Finnish patient.

No association between any of studied haplotypes or microsatellite markers and antibodies against 21-hydroxylase or type 2 autoimmune polyendocrinopathy syndrome was revealed among patients.

\section{Discussion}

Our results confirm the association of both DQB1*02DQA1*05 (DR3-DQ2) and DQB1*0302-(DQA1*03)DRB1*0404 haplotypes with Addison's disease but there were remarkable differences between the populations. DR3-DQ2 association was very strong in the Finnish population, whereas the effect of the DRB ${ }^{*} 0404$ association was in fact stronger among Estonians and Russians when estimated by odds ratio.

These differences could not only be related to patient selection, but might also reflect genetic differences between two more mixed populations and the 'outlier' Finnish population characterized by genetic isolation and strong founder effects. Susceptibility gene alleles associated with Addison's disease might therefore also be more diverse in Estonians and Russians than among Finns. The difference in the frequency of the DR3 haplotype between Finnish and Russian patients cannot be explained by the frequency in the background populations as these are similar.
Haplotype analysis using microsatellite markers did not provide statistical support to the importance of HLA regions other than HLA-DR-DQ loci. However, we found that the HLA-DR3-DQ2 haplotype appears more conserved in Finns and Estonians, than among Russians.

MICA5.1 allele was very common among patients. In the Finnish cases, it was found in 11 of 14 cases but this is the same percentage as was positive for $\mathrm{DQB} 1 * 02-$ DQA $1 * 05$ combination and the allele is in strong linkage with these DQ alleles and also found in more than $50 \%$ of random selection of haplotypes. Results of Estonian patients might be interpreted to support the role of MICA5.1 allele, as this was found in all but two cases. However, among Russians it was again found in about half of the DR3-negative ones and the linkage of it with DR-DQ was not particularly strong in DR3 haplotypes.

The diversity of DRB1*0404-DQB1*0302-positive haplotypes and the fact that DRB1*0401-DQB1*0302 haplotype does not show any association with the disease emphasizes the role of the DRB ${ }^{*} 0404$ molecule itself. In this respect, it is very interesting that DRB ${ }^{*} 0403$ was found increased among Russian patients. It was not found among Estonians and Finns but the frequency of DRB1*0403 is very low among these populations and only DRB1*0401 and DRB1*0404 are the common DR4 alleles found in DR4-DQ8 haplotypes. This is also the case in most populations where DRB $1 * 0404$ association of Addison's disease has been described $(8,9)$. Because families were not available, the origin of each allele cannot be deduced by certainty, but exclusion of the presence of markers in the haplotype can be done.

More data should be collected in populations where DRB1*0403 and/or the structurally similar allele DRB $1{ }^{*} 0406$ as well as other DRB1 alleles *0402 and *0405 are common to test which of the DR4 subtypes in fact show a disease association. The identification of three DQB $1 * 0305$ alleles combined with DRB $1 * 0403$ was also intriguing. This haplotype has been mainly reported in individuals from Sardinia but there is probably a reporting bias as it is not necessarily detected by traditional probe panels (19).

In conclusion, for the first time, we found evidence that the HLA-DRB ${ }^{*} 0403$ allele confers susceptibility to Addison's disease. Additionally, we propose that on the DRB1*0404-DQB1*0302 haplotype the DRB1*0404 allele is more important in conferring disease susceptibility than the DQB1 allele. We found no evidence of a non-class II HLA disease susceptibility locus for Addison's disease; however, the HLA-DR3DQ2 haplotype appeared more conserved in patient groups with high DR-DQ2 frequencies.

\section{Acknowledgements}

We would thank Mare Suigom, Toomas Podar and Ahti Kallikorm for their kind cooperation. 


\section{References}

1 Oelkers W. Adrenal insufficiency. New England Journal of Medicine 1996335 1206-1212.

2 Krohn K, Uibo R, Aavik E, Peterson P \& Savilahti K. Identification by molecular cloning of an autoantigen associated with Addison's disease as steroid 17a-hydroxylase. Lancet 1992339 770-773.

3 Winqvist O, Karlsson FA \& Kampe O. 21-Hydroxylase, a major autoantigen in idiopathic Addison's disease. Lancet 1992339 1559-1562.

4 Falorni A, Nikoshkov A, Laureti S, Grenback E, Hulting AL, Casucci G, Santeusanio F, Brunetti P, Luthman H \& Lernmark A. High diagnostic accuracy for idiopathic Addison's disease with a sensitive radiobinding assay for autoantibodies against recombinant human 21-hydroxylase. Journal of Clinical Endocrinology and Metabolism 199580 2752-2755.

5 Neufeld M, Maclaren NK \& Blizzard RM. Two types of autoimmune Addison's disease associated with different polyglandular autoimmune (PGA) syndromes. Medicine 198160 355-362.

6 Thomsen M, Platz P, Andersen OO, Christy M, Lyngsooe, Nerup J, Rasmussen K, Ryder LP, Nielsen LS \& Svejgaard A. MLC typing in juvenile diabetes mellitus and idiopathic Addison's disease. Transplantation Reviews 197522 125-147.

7 Maclaren NK \& Riley WJ. Inherited susceptibility to autoimmune Addison's disease is linked to human leukocyte antigens-DR3 and/or DR4, except when associated with type I autoimmune polyglandular syndrome. Journal of Clinical Endocrinology and Metabolism $1986 \mathbf{6 2} 455-459$.

8 Myhre AG, Undlien DE, Lovas K, Uhlving S, Nedrebo BG, Fougner KJ, Trovik T, Sorheim JI \& Husebye ES. Autoimmune adrenocortical failure in Norway autoantibodies and human leukocyte antigen class II associations related to clinical features. Journal of Clinical Endocrinology and Metabolism 200287 618-623.

9 Yu L, Brewer KW, Gates S, Wu A, Wang T, Babu SR, Gottlieb PA, Freed BM, Noble J, Erlich HA, Rewers MJ \& Eisenbarth GS. DRB ${ }^{*} 04$ and DQ alleles: expression of 21-hydroxylase autoantibodies and risk of progression to Addison's disease. Journal of Clinical Endocrinology and Metabolism 199984 328-335.

10 Park YS, Sanjeevi CB, Robles D, Yu L, Rewers M, Gottlieb PA, Fain P \& Eisenbarth GS. Additional association of intra-MHC genes, MICA and D6S273, with Addison's disease. Tissue Antigens 200260 155-163.

11 Bilbao JR, Martin-Pagola A, Perez De Nanclares G, Calvo B, Vitoria JC, Vazquez F \& Castano L. HLA-DRB1 and MICA in autoimmunity: common associated alleles in autoimmune disorders. Annals of the New York Academy of Sciences 20031005 314-318.

12 Gambelunghe G, Falorni A, Ghaderi M, Laureti S, Tortoioli C, Santeusanio F, Brunetti P \& Sanjeevi CB. Microsatellite polymorphism of the MHC class I chain-related (MIC-A and MIC-B) genes marks the risk for autoimmune Addison's disease. Journal of Clinical Endocrinology and Metabolism 199984 3701-3707.

13 Barker JM, Ide A, Hostetler C, Yu L, Miao D, Fain PR, Eisenbarth GS \& Gottlieb PA. Endocrine and immunogenetic testing in individuals with type 1 diabetes and 21-hydroxylase autoantibodies: Addison's disease in a high-risk population. Journal of Clinical Endocrinology and Metabolism 200590 128-134.

14 Peterson P, Uibo R, Peranen J \& Krohn K. Immunoprecipitation of steroidogenic enzyme autoantigens with autoimmune polyglandular syndrome type I (APS I) sera: further evidence for independent humoral immunity to $\mathrm{P} 450 \mathrm{c} 17$ and P450c21. Clinical and Experimental Immunology 1997107 335-340.

15 Nejentsev S, Koskinen S, Sjoroos M, Reijonen H, Schwartz EI, Kovalchuk L, Sochnev A, Adojaan B, Podar T, Knip M, Simell O, Koskenvuo M, Akerblom HK \& Ilonen J. Distribution of insulindependent diabetes mellitus (IDDM)-related HLA alleles correlates with the difference in IDDM incidence in four populations of the Eastern Baltic region. Tissue Antigens 199852 473-477.

16 Hermann R, Turpeinen H, Laine AP, Veijola R, Knip M, Simell O, Sipila I, Akerblom HK \& Ilonen J. HLA DR-DQ-encoded genetic determinants of childhood-onset type 1 diabetes in Finland: an analysis of 622 nuclear families. Tissue Antigens $2003 \mathbf{6 2}$ $162-169$.

17 Foissac A \& Cambon-Thomsen A. Microsatellites in the HLA region: 1998 update. Tissue Antigens 199852 318-352.

18 Nejentsev S, Reijonen H, Adojaan B, Kovalchuk L, Sochnevs A, Schwartz EI, Åkerblom HK \& Ilonen J. The effect of HLA-B allele on the IDDM risk defined by DRB1*04 subtypes and DQB1*0302. Diabetes 199746 1888-1892.

19 Cucca F, Frau F, Lampis R, Floris M, Argiolas L, Macis D, Cao A, Devirgiliis S \& Congia M. HLA-DQB1*0305 and -DQB1*0304 alleles among Sardinians - evolutionary and practical implications for oligotyping. Human Immunology 199440 143-149.

Received 6 September 2007

Accepted 12 September 2007 